\title{
Photocatalysis for Water Treatment: A Sustainable Sanitation Access in Daily Life
}

\author{
Chanchal Hazra \\ INRS-EMT, University of Quebec, Canada \\ E-mail: chanchalhazra007@gmail.com
}

https://doi.org/10.54162/SD01-25201/06

Read online Cite this: Sc.Dialectica, 2021, vol. 01, pp. 19- 21

Keywords: Photocatalysis, Waste Water Treatment, $\mathrm{TiO}_{2}$

\begin{abstract}
Enormous technological progress and widespread industrialization has made human life easier but at the cost of imbalance in our biosphere. Anthropogenic activities during the last century have engendered serious environmental issues including water pollution. Huge amount of a variety of toxic organic (synthetic dyes, antibiotics, pesticides) and inorganic (heavy metal ions) pollutants are constantly being discharged as agricultural runoffs or as industrial wastes from textiles, leather, cosmetic, paper, dyeing and printing industries into lakes, streams, rivers, oceans, and other water bodies thereby deteriorating water quality and adversely affecting both aquatic and human life.
\end{abstract}

\section{Introduction}

In India, contamination of fresh water bodies by highly toxic and carcinogenic agrochemical from agriculture fields and heavy metals (arsenic (III), chromium (VI)) from Himalayan ore mines has become a major health threat affecting the lives of around 0.050 billion people. According to the WHO's 2017 report, approximately 844 million people worldwide still lack access to clean drinking water and the situation becomes worst in case of floods and earthquakes.[1] Water-borne diseases associated with the consumption of polluted water have become a serious health challenge and millions of peoples are reported to die due to serious waterborne diseases.

\section{Discussion}

To address the problems of water pollutants, researchers and scientists have been striving to develop new methods and/or improve the existing classical methods of waste-water treatment (Scheme 1). Conventional methods of wastewater treatment including chlorination, flocculation, and adsorption have been used since long but they are limited in their capacity to completely remove toxic pollutants. For instance, chlorination is effective to kill microorganisms but cannot remove organic and inorganic pollutants from water. Similarly, using some adsorbent materials to adsorb pollutants from water is effective in removing certain pollutants but they lack the capacity to completely degrade them. Likewise, membrane technologies have found good use in treatment of wastewater but the applicability of this technology is still hindered by the issue of membrane fouling. In this scenario, advanced oxidation processes (AOPs) have emerged as a promising solution for complete removal of both organic and inorganic pollutants from water.

Among these AOPs, photocatalysis, ozone treatment (ozonation) and electrochemical processes or a combination of these processes have attracted great attention for their use in water treatment. In the ozonation process, ozone, a highly oxidizing gas, is introduced into wastewater which directly or indirectly interacts with particles present in the wastewater leading to the decomposition of pollutants. Similarly, hydrogen peroxide together with iron (Fe(II)) salts, can be added to water to generate reactive oxidant species capable of degrading pollutants in a process known as Fenton oxidation process.

Of greater value among AOPs is heterogeneous photocatalysis which employs certain photoactive compounds known as photocatalysts and light to detoxify water and create safe drinking water. Common examples of such materials are titanium dioxide $\left(\mathrm{TiO}_{2}\right)$, zinc oxide $(\mathrm{ZnO})$, bismuth vanadate $\left(\mathrm{BiVO}_{4}\right)$, among others.[2,3] In the presence of suitable light (such as UV light or normal solar light) and humidity, these photocatalysts have the ability to produce highly reactive oxidant species (such as superoxide and hydroxyl radicals) which are capable of fully destroying organic pollutants (often converting them into water and carbon dioxide) and kill microorganism presents in water (Scheme 2). Similarly, the interaction of photocatalysts with light (known as photoexcitation) leads 


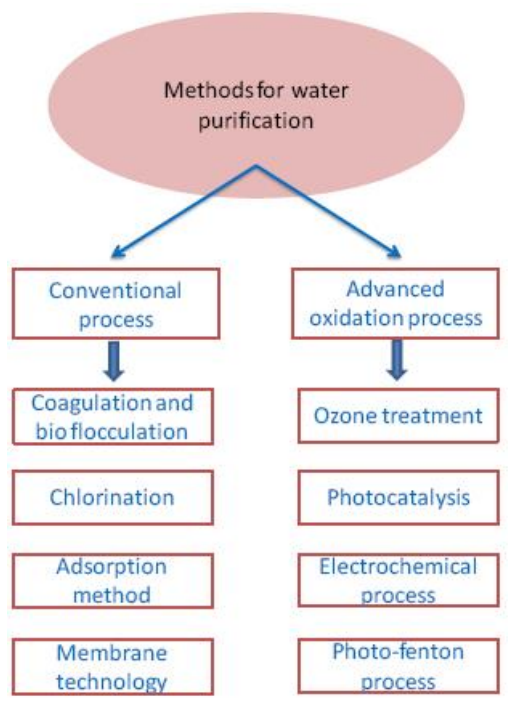

Scheme 1

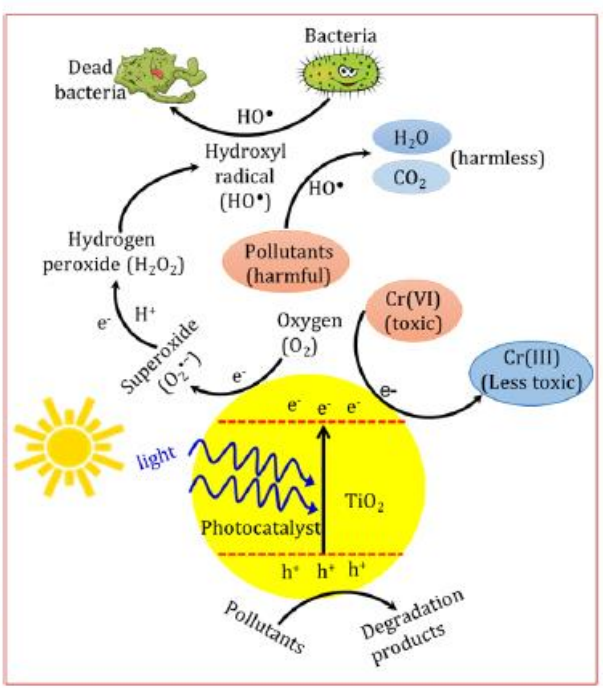

Scheme 2

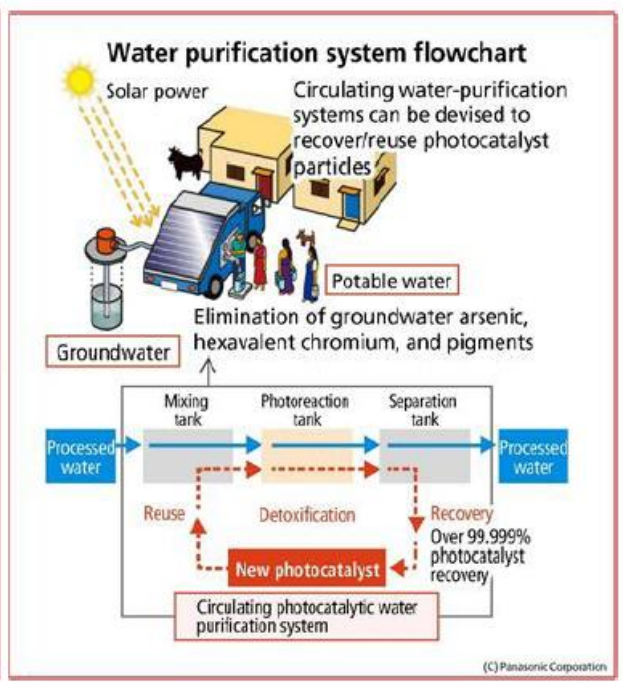

Scheme 3

Scheme 1. Selected water-purification methods. Scheme 2. Different processes occurring during heterogeneous photocatalysis that ultimately lead to the formation of reactive species which take part in degradation of pollutants and killing of microbes. Scheme 3. Panasonic's photocatalytic water purification system. ${ }^{[4]}$

to the formation of certain vacancies (known as holes, $\mathrm{h}^{+}$) due to loss/excitation of electron $\left(\mathrm{e}^{-}\right)$to higher energy levels. These holes are oxidizing in nature and extract electrons from pollutant molecules and degrade them in the process. Moreover, these photogenerated oxidizing species have a shorter lifetime and self-eliminate during their degradation of pollutants.

The semiconductor photocatalysts-based photocatalytic process has gained much attention not only in the field of wastewater treatment but also energy generation using the abundantly available natural sunlight. Since the artificial UV sources used in photocatalysis make the photocatalytic water treatment process expensive, attempts are being made to prepare photocatalyst able to harvest natural sunlight for water purification and energy generation. Since photocatalysts usually consist of extremely small (nanometer or micrometer regime) particles, it becomes difficult to separate them from water after being used. Thus an important issue with the use of photocatalysts for water purification is their recycling and separation from water after use. This problem is often overcome by supporting the fine photocatalysts particles on some inert substrate (glass, polymer etc.) in the form of thin film. Doing so, however, decreases the exposed surface area and hence water purification efficiency. Moreover, despite the great promise photocatalysis holds to detoxify polluted water and safe and drinkable water, commercial application of the process have only recently begun.[4,5] For instance, Panasonic has proposed a very efficient photocatalytic system based on titanium dioxide/zeolite photocatalysts for water purification[4] (Scheme 3). Though small scale photocatalytic water purification systems are already commercially available [5], further efforts are required to extend the technology toward high-volume photocatalytic water treatment plants.

\section{References}

[1] A. P. Ustün et al. "Burden of disease from inadequate water, sanitation and hygiene in low- and middle-income settings: a retrospective analysis of data from 145 countries", Tropical Medicine and International Health, 2014, vol. 19, pp. 894-905.

[2] M. J. Wu, T. Bak, P. J. O’Doherty, M. C. Moffitt, J. Nowotny,T. D. Bailey, and C. Kersaitis, "Photocatalysis of Titanium Dioxide for Water Disinfection: Challenges and Future Perspectives", International Journal of Photochemistry, 2014, vol. 2014, pp. 1-9.

[3] S. Ullah, E.P. Ferreira-Neto, C. Hazra, R. Parveen, H.D. Rojas-Mantilla, M. L. Calegaro, Y.S. Correales, U. P. Rodrigues-Filho, S. J. L. Ribeiro, "Broad spectrum photocatalytic system based on $\mathrm{BiVO}_{4}$ and $\mathrm{NaYbF}_{4}: \mathrm{Tm}^{3+}$ upconversion particles for environmental remediation under UV-vis-NIR illumination", Appl. Catal. B-Environ., 2019, vol. 243, pp. 121-135.

[4] "Photocatalytic water purification technology", https://news.panasonic.com/global/stories/2014/30520.ht $\mathrm{ml}$. 
[5] S. K. Loeb et al., "The Technology Horizon for Photocatalytic Water Treatment: Sunrise or Sunset?" Environ. Sci. Technol. 2019, vol. 53, pp. 2937-2947.

Author's biography

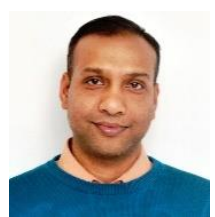

Dr. Chanchal Hazra is Postdoctoral Research Fellow in Chemistry at the National Institute of Scientific Research - Energy Materials and Telecommunication (INRS-EMT), University of Quebec, QC, Canada. He received his $\mathrm{PhD}$ in Chemical Sciences at the Indian Institute of Science Education and Research (IISER)-Kolkata, India in 2015. He worked as Postdoctoral Research Fellow at the Institute of Chemistry, State University of Sao Paulo (IQ-UNESP), SP-Brazil. Dr. Hazra's research interest encompasses three distinct areas namely upconverting/downshifting nano/micromaterials based on rare earth (often called lanthanide) ions, novel photoactive materials and multifunctional nanoplatforms for applications in heavy metals/organic molecules sensing, environmental remediation as well potential applications in biomedical direction. 